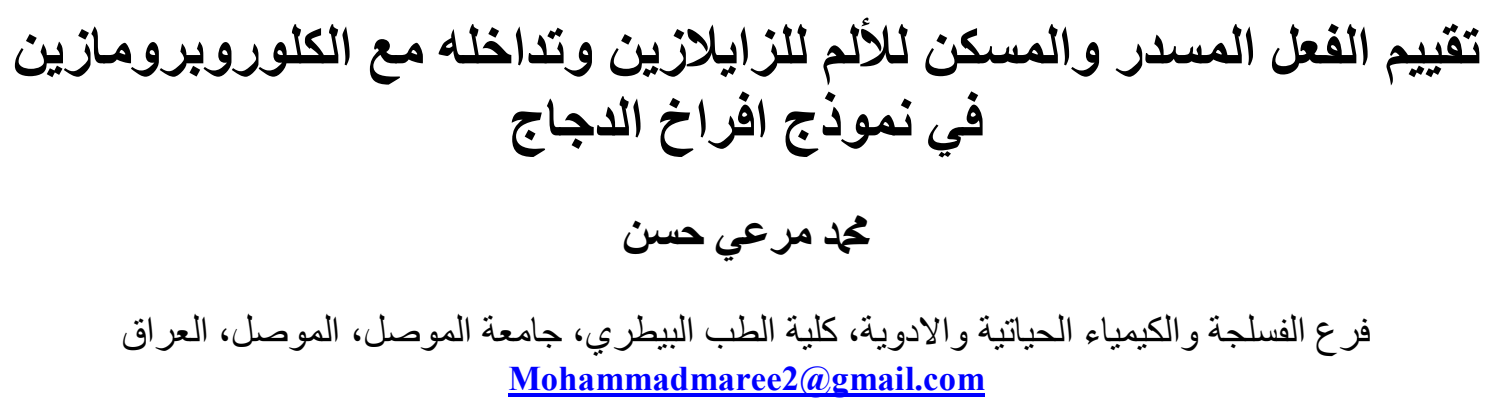

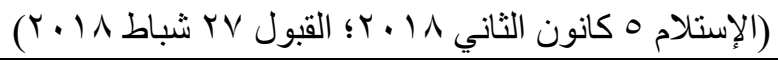

استهدفت الدراسة تقييم الفعل المسدر للألم للزايلازين لوحده او عند اعطائه مع الكلوروبرومازين الكازين وفحص التسكين من الالم باستخدام

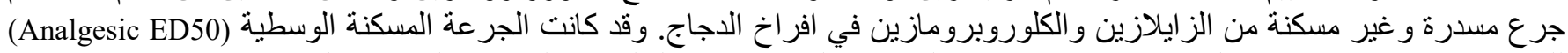

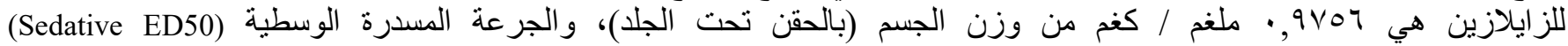

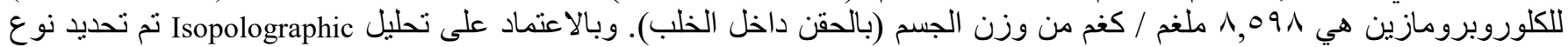

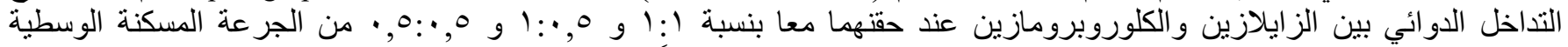

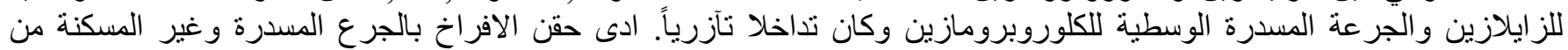

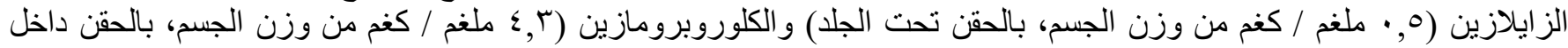

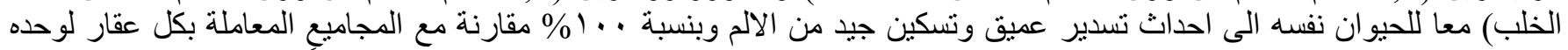

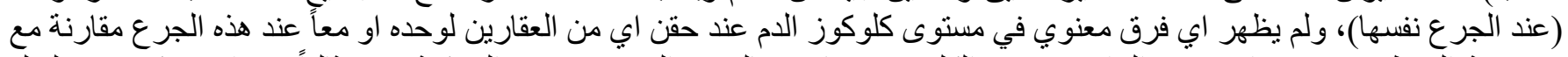

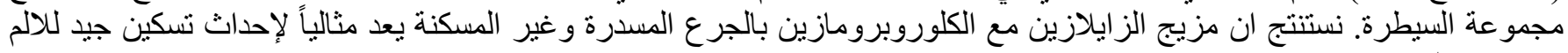

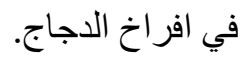

\title{
Evaluating the sedative and analgesic effects of xylazine and it's interaction with chloropromazine in chicks
}

\author{
M.M. Hasan \\ Department of Physiology, Biochemistry \& Pharmacology, College of Veterinary Medicine, University of Mosul, Mosul, Iraq
}

\begin{abstract}
The aim of this study was to evaluate the sedative effect of xylazine alone or as a combination with chloropromazine, and to check the possibility to induce analgesia by the use of sedative (sub analgesic) doses of this combination in chicks. The analgesic $\mathrm{ED}_{50}$ of xylazine (S.C.) and chloropromazine (I.P.) was 0.9756 and $8.598 \mathrm{mg} / \mathrm{kg}$ respectively. Depending on Isopolographic analysis, the drug interaction between xylazine and chloropromazine at ratios of 1:1, 0.5:1 and 0.5:0.5 of the analgesic $\mathrm{ED}_{50}$ of xylazine and the sedative $\mathrm{ED}_{50}$ of chloropromazine was synergistic interaction. The injection of sedative (non - analgesic) doses of xylazine ( $0.5 \mathrm{mg} / \mathrm{kg}$ body weight, S.C.) and chloropromazine ( $4.3 \mathrm{mg} / \mathrm{kg}$ body weight, I.P.) together induced deep sedation with excellent analgesia in $100 \%$ of the animals in comparison with each drug alone at the same doses. There were no significant differences in glucose levels between the group of xylazine $(0.5 \mathrm{mg} / \mathrm{kg}$ body weight, S.C.), a group of chloropromazine $(4.3 \mathrm{mg} / \mathrm{kg}$ body weight, I.P.) or group of xylazine $(0.5 \mathrm{mg} / \mathrm{kg}$ body weight, S.C.) and chloropromazine $(4.3 \mathrm{mg} / \mathrm{kg}$ body weight, I.P.) together compared with the control group. Therefore, the combination sedative doses of xylazine and chloropromazine (low doses) can be considered as an ideal mixture for good analgesia in chicks.
\end{abstract}

Available online at http://www.vetmedmosul.org/ijvs 


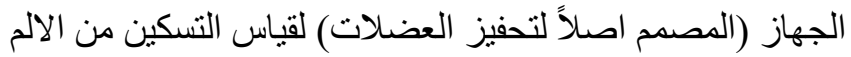

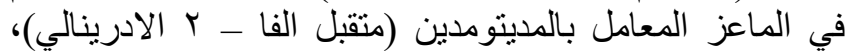

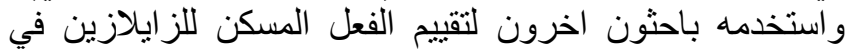

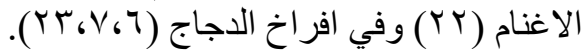

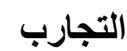

تحديد الجرعة المسكنة الوسطية (الجرعة الفاعلة الوسطية

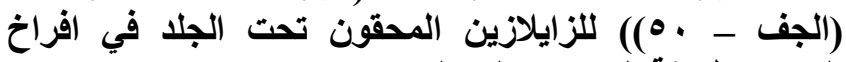
الدجاج بطريقة الصعود والنزول النزول

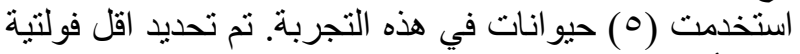
مسببة للألم (الصياح) للحيوان الاول باستخدام جهاز المحفز

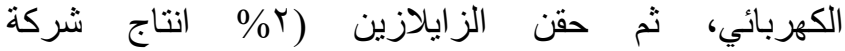
وزalfasanwoerden

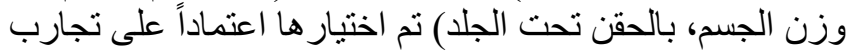

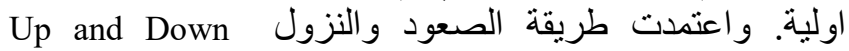
وكان Method

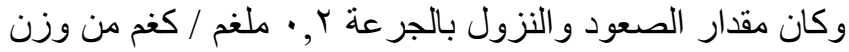

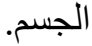

تحديد الجرعة المسدرة الوسطية (الجرعة الفاعلة الوسطية

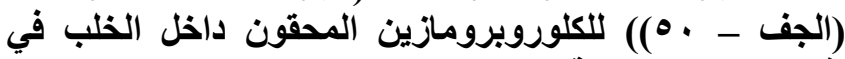

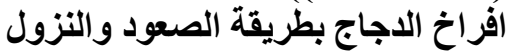

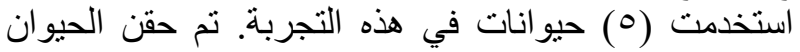

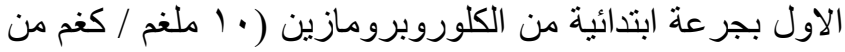

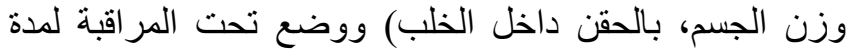

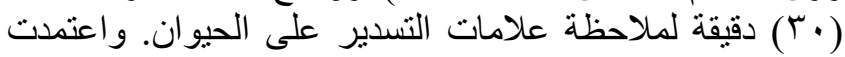

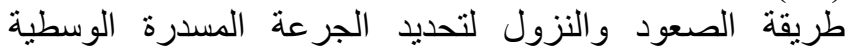

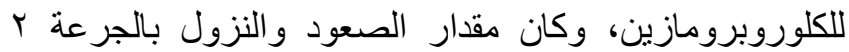

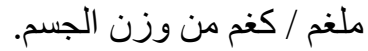

تحديد نوع التداخل الدوائي بين الزايلازين المحقون تحت الجلد

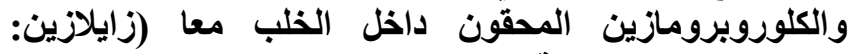

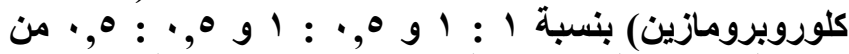

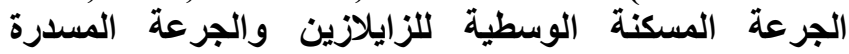

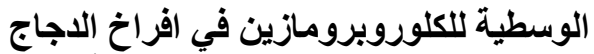

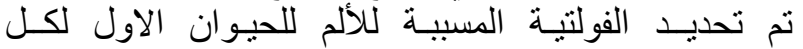

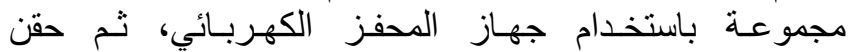

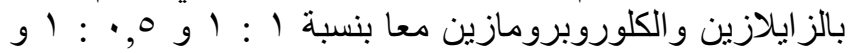

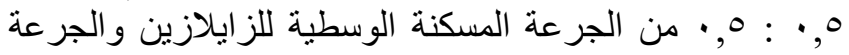

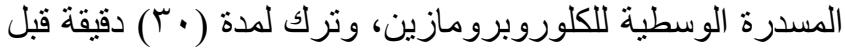

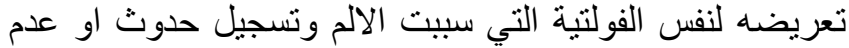

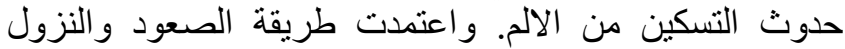

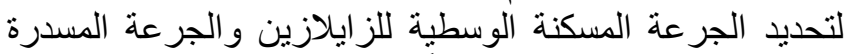

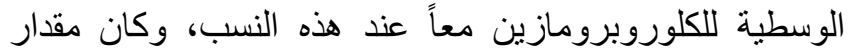

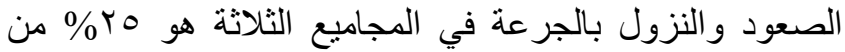

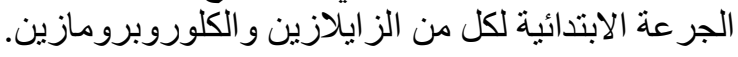

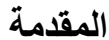

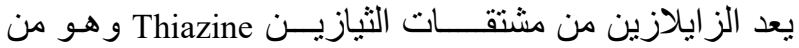

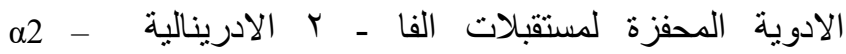
الإن adrenoceptor agonist

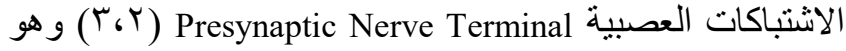
يستخدم بشكل واسع في العديد من الحيوانات الحقلية كالاجاج

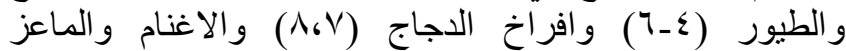

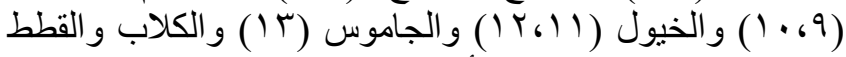

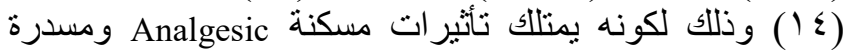

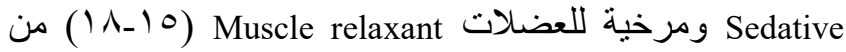

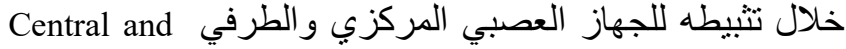
- عن طريق تحفيز مستقبلات الفاكئ

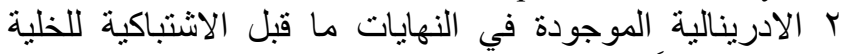

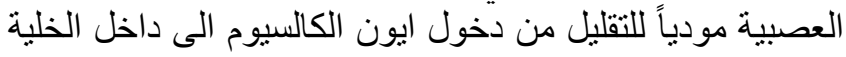

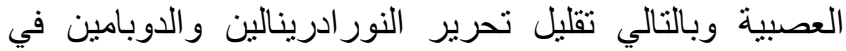

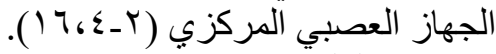

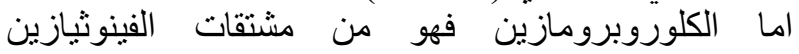

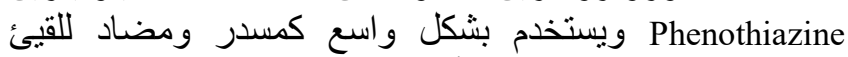

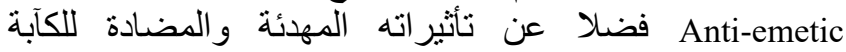

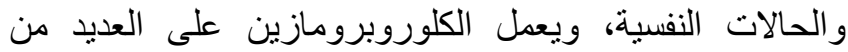

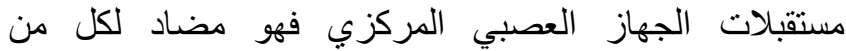
Anti-dopaminergic, مستقبلات الدوبامين والهستامين والكولين فئين anti-histaminergic and anti-cholinergic

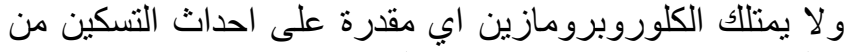

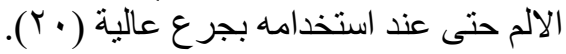

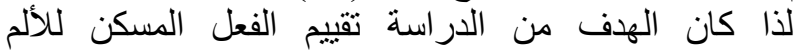

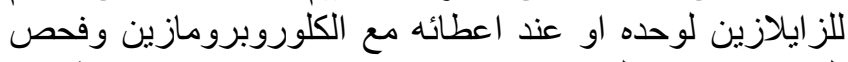

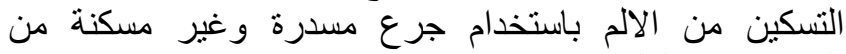

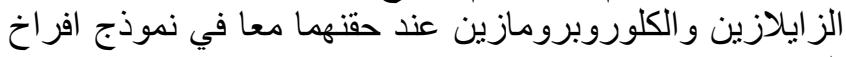

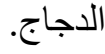

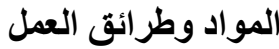

اجريت الدراسة في كلية الطب البيطري / جامعة الموصل

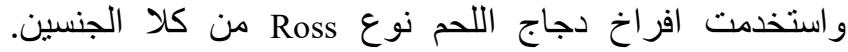

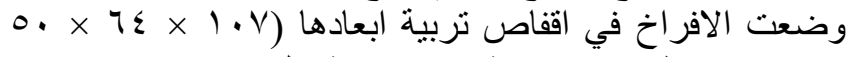

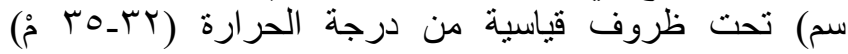

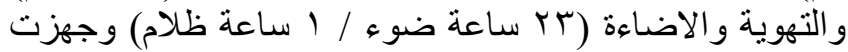

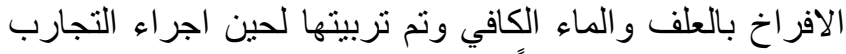

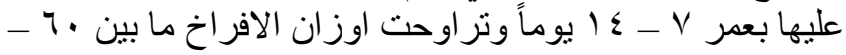

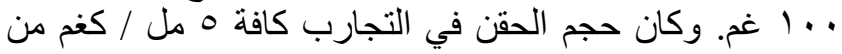

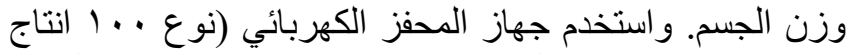

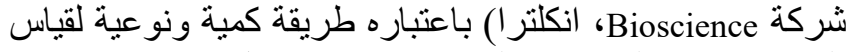
التسكين من الالم، ويعد المشهداني (Yl) اول من استخدم هذا 
التحليل الاحصائي تم تحليل نتائج البيانات المخبرية Parametric Data إحصائيـا باستخدام تحليـل التبايـ One Way analysis of variance

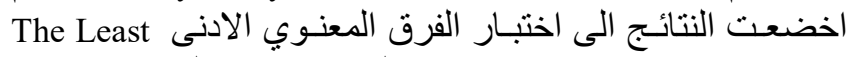
Non- Significant Test

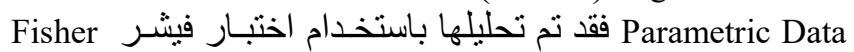
Exact Probability للتجارب كافة عند مستوى احتمال (أ > 0 •, • ).

النتائج

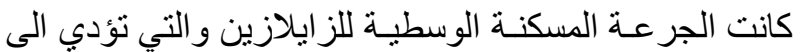

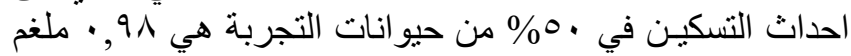

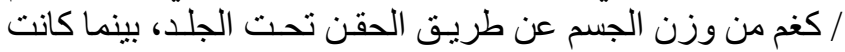
الجرعة المسدرة الوسطية للكلوروبرومازين المحقون داخل داخل الخلب هي 1, 1 ملغم / كغم من وزن الجسم (الجدول ( ). وتمثلت

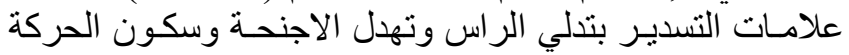

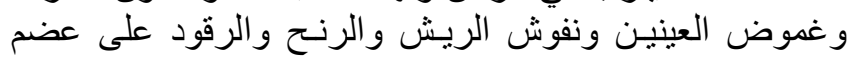
وكانت الجرعة المسكنة الوسطية للزايلازين و الجرعة المسدرة الوسطية للكلوروبرومازين عند حقن العقارين معا

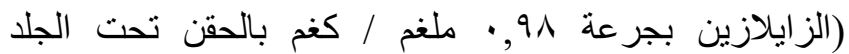

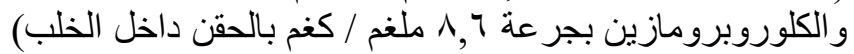

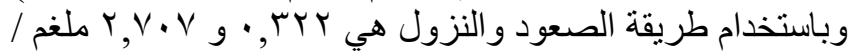

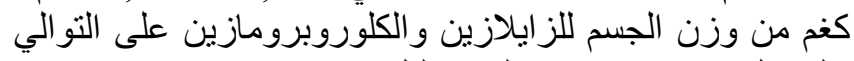

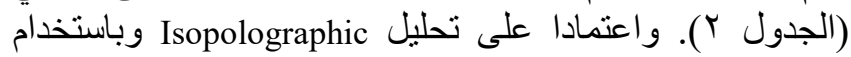
نسب مختلفة من الجرعة المسكنة الوسطية للز ايلازين و الجرعة

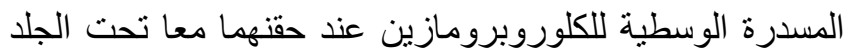

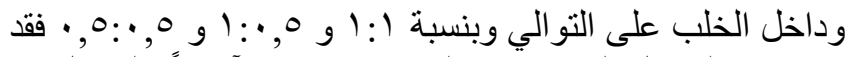

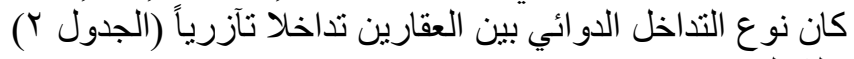

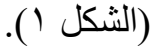

استخدام جرع مسدرة وغير مسكنة من الزايلازين او غير

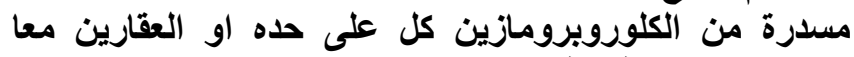
لإحداث التسكين في افراخ الاجداج قسمت الافراخ في هذه التجربة فئ على أربع مجاميع منفصلة

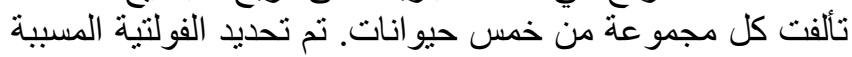
للألم لحيوانات كل مجموعة باستخدام جهاز المحفز الكهربائي، ثم حقتت حيوانات المجموعة الأولى (السيطرة) بالمحلول الملحي

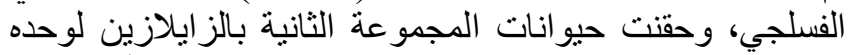

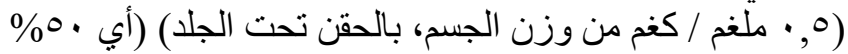
من الجرعة المسكنة الوسطية للزايلازين)، في حين حفن حقنت حيوانات المجمو عة الثالثة بالكلوروبرومازين لوحده (ب, كـ ملغم /

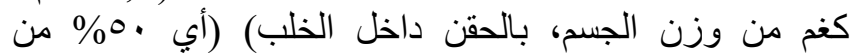

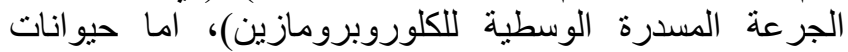

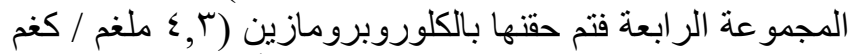
من وزن الجسم، بالحقن داخل الخلب) ثم أعبد حقنها مباشرة

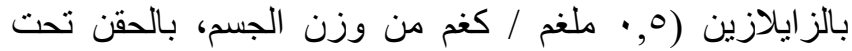
الجلد)، وبعد (·) (ب) دقيقة تم تعريض الحيوانات في المجاميع الاربعة للفولتية نفسها التي سببت الالم وتسجيل حدوث دئث او عدم حدوث التسكين.

تأثير استخدام الجرع المسدرة وغير المسكنة من الزايلازين او اوني

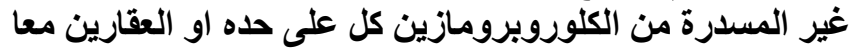

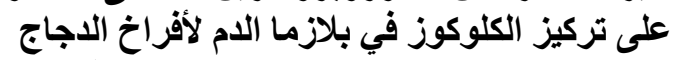

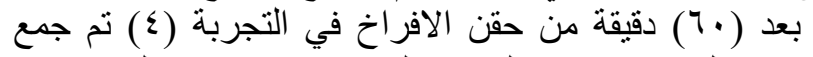

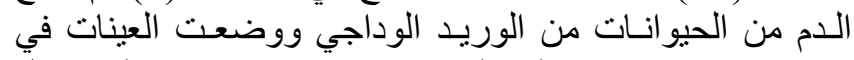

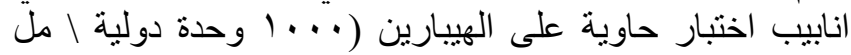

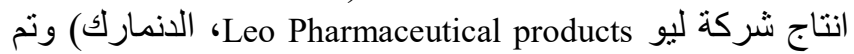
فصل البلازما باستخدام جهاز الطرد المركزي (إنتاج شركة فئمان

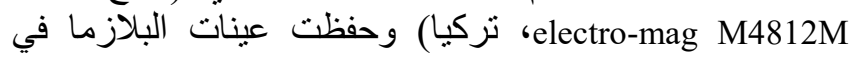

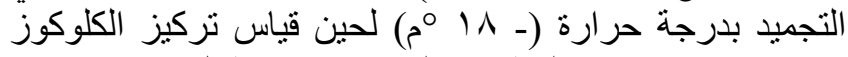
فيها باستخدام جهاز المطياف الضونة مائي عند طول موجي

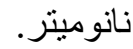

الجدول ا: تحديد الجرعة المسكنة الوسطية للزايلازين بالحقن تحت الجلد و الجرعة المسدرة الوسطية للكلوروبرومازين بالحقن داخل الخلب في افر اخ الاجاج

\begin{tabular}{|c|c|c|}
\hline الكلوروبرومازين & الز ايلازين & القياسات \\
\hline ........... & 9V0T, 9 • ملغم / كغم & الجرعة المسكنة الوسطية \\
\hline 1,091 ملغم / كغم & & الجرعة المسدرة الوسطية \\
\hline 1 - · ا ملغم / كغم & ^, • - I, 1 ملغم / كغم & مدى الجرع \\
\hline ا ملغم / كغم & r, ملغم / كغم & الجر عة الأولية \\
\hline ا. ا ملغم / كغم & ^, • ملغم / كغم & الجرعة النهائية \\
\hline r ا ملغم / كغ & r r • ملغم / كغم & مقدار الزيادة او النقصان في الجرعة \\
\hline (XOXOX) 5 & (XOXXO) 5 & عدد الأفر اخ المستخدمة \\
\hline
\end{tabular}


الجدول ؟: تحديد الجر عة المسكنة الوسطية للزايلازين و الجر عة المسدرة الوسطية للكلوروبرومازين عند حقهما معاً بنسب مختلفة

\begin{tabular}{|c|c|c|c|}
\hline \multicolumn{3}{|c|}{ الز ايلازين : الكلوروبرومازين } & \multirow{2}{*}{ 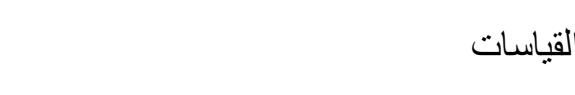 } \\
\hline$, 0:, 0$ & $1: \cdot, 0$ & $1: 1$ & \\
\hline ا & 17, • ملغم / كغم & 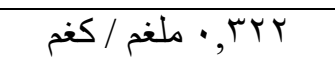 & الجر عة المسكنة الوسطية للز ايلازين \\
\hline O, • - 0, • ملغم/كغ & 0ץ ו, • - 0, • ملغم/كغم & ד r, • - & \\
\hline 0, • ملغم / كغم & مْ , • ملغم / كغم & | 91,9 • ملغم / كغم & الجر عة الأولية \\
\hline 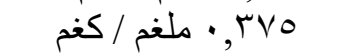 & O T, • ملغم / كغم & T ب, • ملغم / كغم & الجرعة النهائية \\
\hline \% \% ( 0 1, • ملغم/كغم) & 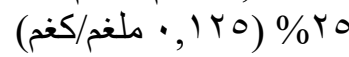 & OY\% \% ( ז, • ملغم/كغم) & مقدار الزيادة او النقصان في الجرعة \\
\hline$(\mathrm{XXOXOX)} 6$ & $(\mathrm{XXXOXOX)} 7$ & (XXXOXOX) 7 & عدد الافر اخ المستخدمة \\
\hline سT گ T ملغم / كغم & 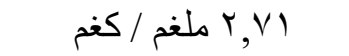 & V ملغم / r,V.V مغم & الجرعة المسدرة الوسطية للكلوروبرومازين \\
\hline 10 & & & 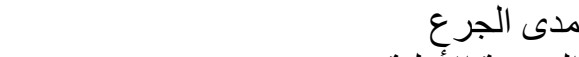 \\
\hline مابرء ملغم / كغم & 1 1, ملغم / كغم & 1, 1, ملغم / كغم & الجر عة الأولية \\
\hline 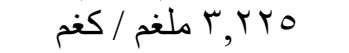 & 10 10 ملغم / كغم & & الجر عة النهائية \\
\hline \% \% (V0, , ا ملغم/كغم) & 0\% \% (0, r ملغم/كغم) & 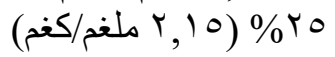 & مقدار الزيادة او النقصان في الجرعة \\
\hline$(\mathrm{XXOXOX)} 6$ & $(\mathrm{XXXOXOX)} 7$ & $(\mathrm{XXXOXOX)} 7$ & عدد الأفر اخ المستخدمة \\
\hline
\end{tabular}

حين نجح اعطاء العقارين معا بالجرع السابقة نفسها في احداث

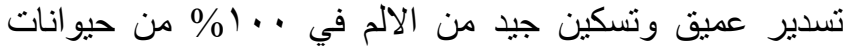

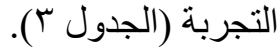

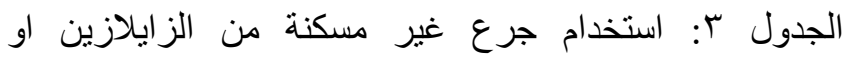
الكلوروبرومازين كل لوحده او العقارين معا لإحداث التسكين في التيلي افر اخ الدجاج

\begin{tabular}{|c|c|c|}
\hline التسكين 0 & 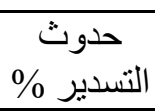 & المجاميع \\
\hline$\cdot$ & $\cdot$ & السبطرة \\
\hline . & 7. & الزايلازين \\
\hline - & - & الكلوروبرومازين \\
\hline$* 1 \ldots$ & $* 1 \ldots$ & والزلوايلازين \\
\hline
\end{tabular}

(*) تعني وجود فرو مازين معنوي مقارنة مع مجموعة السيطرة

. $(\mathrm{P}<\cdot, \cdot 0)$

واظهرت النتائج عدم وجود اي تغيير معنوي في تركيز

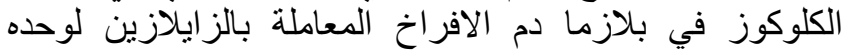

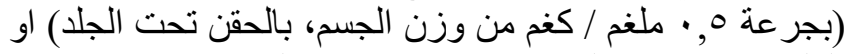

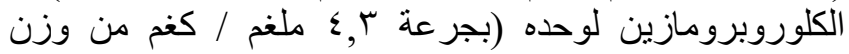

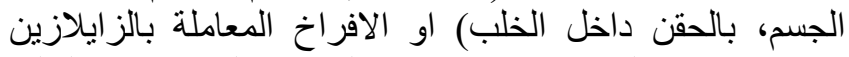

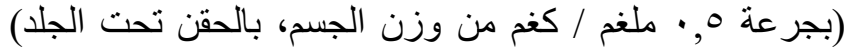

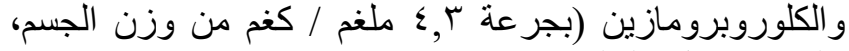

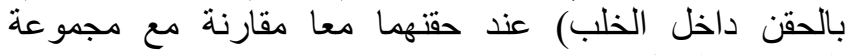

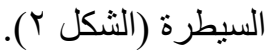

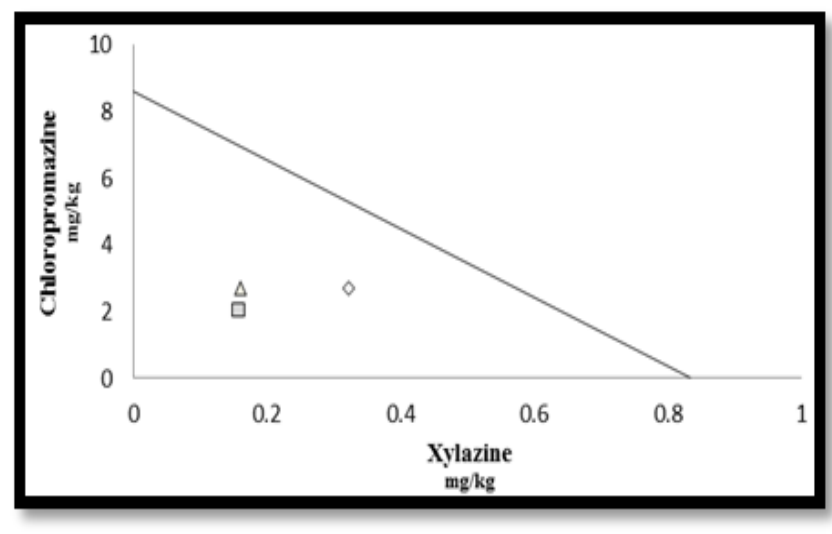

الثكل 1: يوضح نوع التداخل الدوائي بين الزايلازين

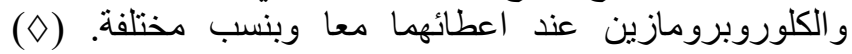

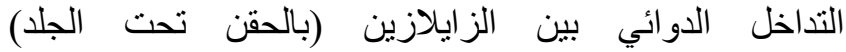

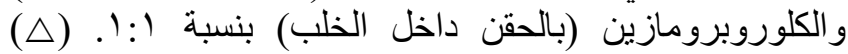

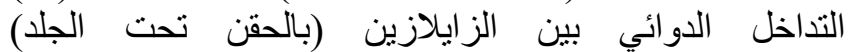

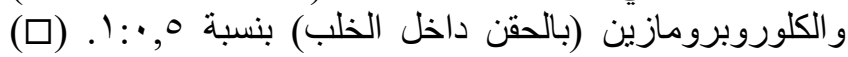

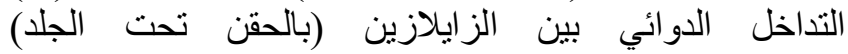

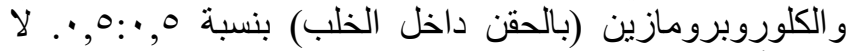
يوجد أي فرق معنوي بين المجاميع (0 حيورانات دات لكل مجموعة)

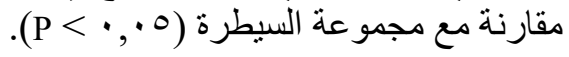

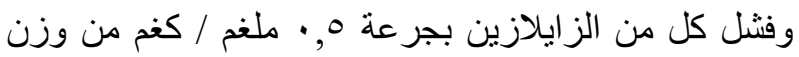

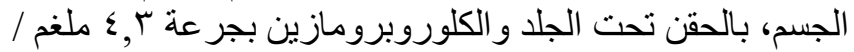

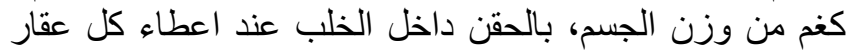
لوحده في احداث اب تسكين من الالم في حيوانات التجربة، في أخل في 
قوي معتمد على الجرعة، لذلك تسمى قاتلات الألم اذ إنها تمنع

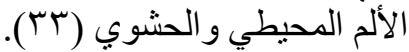

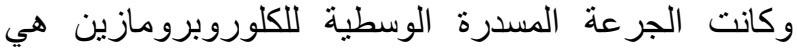

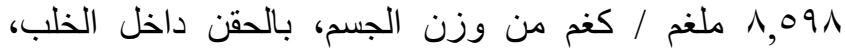

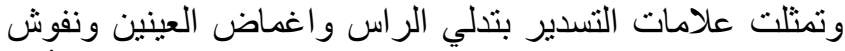

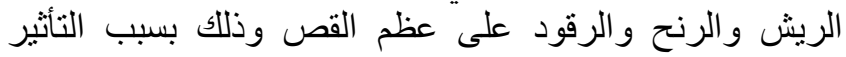

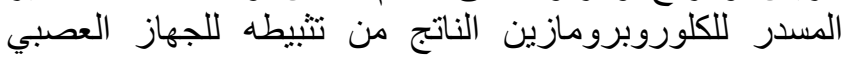

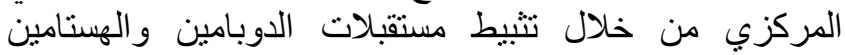

اما حقن الزايلازين والكلوروبرومازين معا وبنسب مختلفة

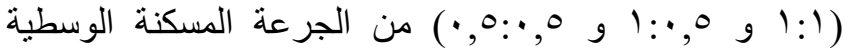

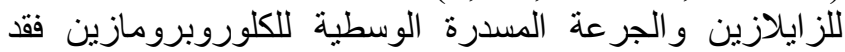

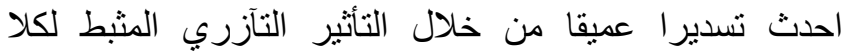

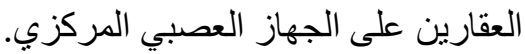

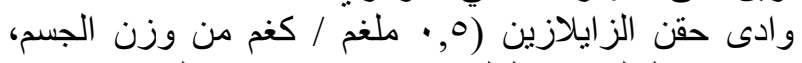

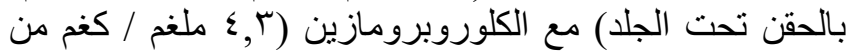

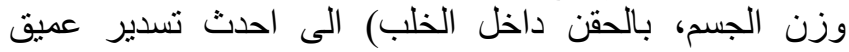

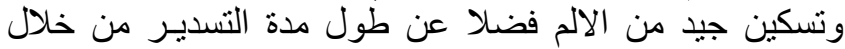

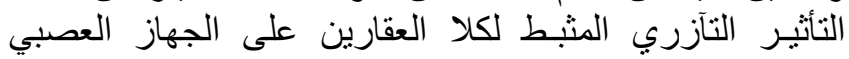

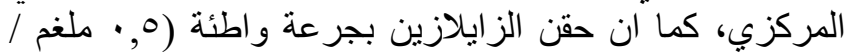

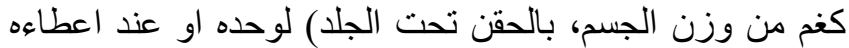

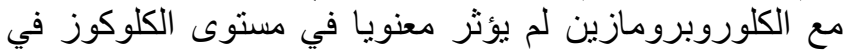

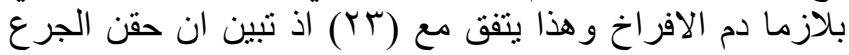

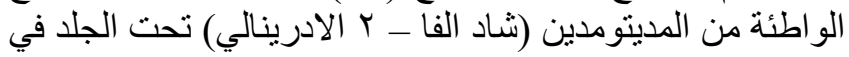

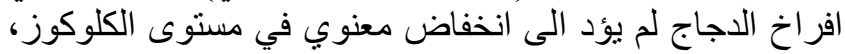

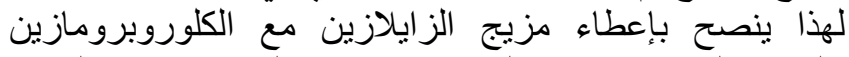
بالجرع المسدرة وغير المسكنة لإحداث التراء التسكين من الالم في

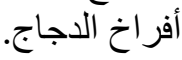

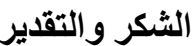

اتقدم بالشكر الجزيل لعمادة كلية الطب البيطري / جامعة

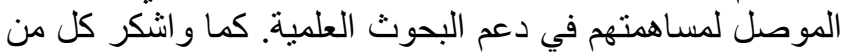

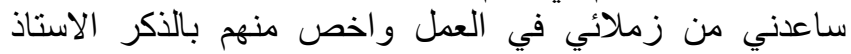
الدكتورة غادة عبد المنعم فارس التي كان لها دور كبي لهيبر في انجاز هذا البحث.

المصادر

1. Adams HR. Veterinary Pharmacology and Therapeutics. $8^{\text {th }}$ ed. Iowa State: University Press; 2001.

2. Kleinz MJ, Spence I. The pharmacology of the autonomic nervous system. In: Madison J. editor. Small animal clinical pharmacology. $2^{\text {nd }}$ ed. Philadelphia: Saunders Elsevier. 2008;pp:59-82.

3. Pawson P. Sedatives. In: Maddison, J. (editor), Small animal clinical pharmacology. $2^{\text {nd }}$ ed., Philadelphia: Saunders Elsevier. 2008;pp:113125 .

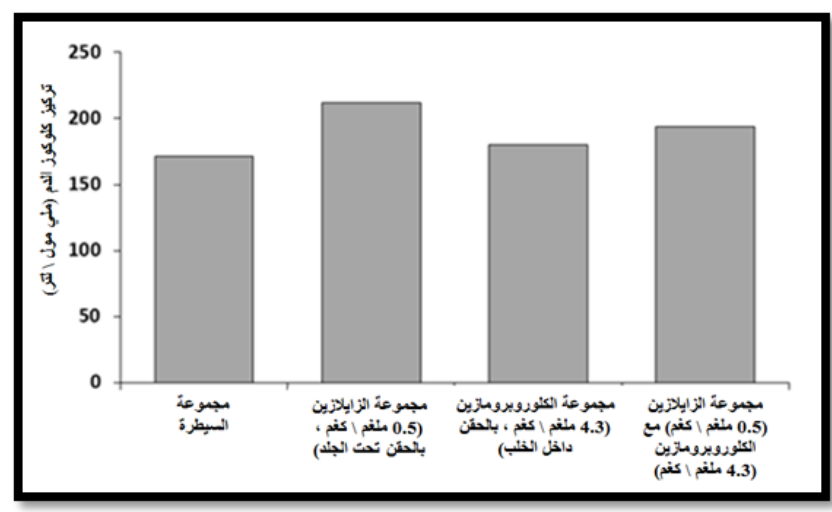

الثكل r ب: يوضح تأثير المعاملة بالز ايلازين والكلوروبرومازين

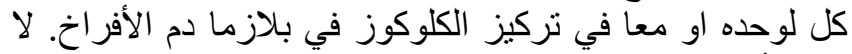

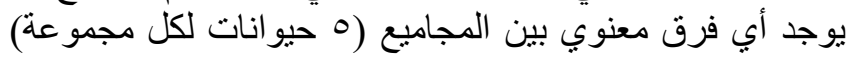

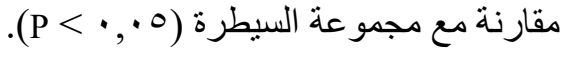

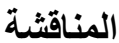

استخدم جهاز المحفز الكهربائي لتقييم الفعل المسكن والمسدر

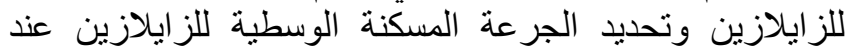

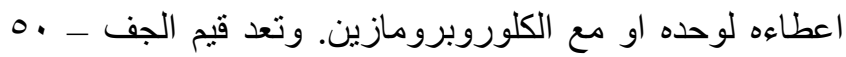

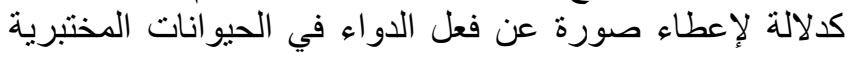

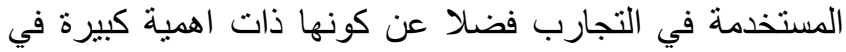

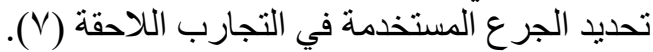

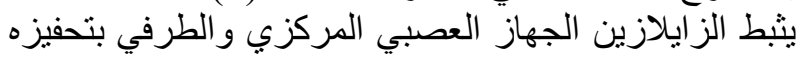

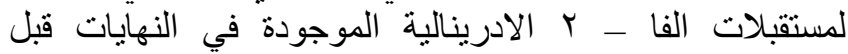
الاشتباكية للخلية العصبية مؤديا للتقليل من دخول الإلية ايون الكالسيوم

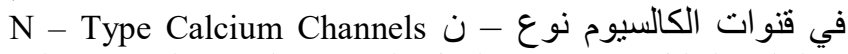
وبالتالي التقليل من افراز الناقل العصبي المهيج النورادرينالين

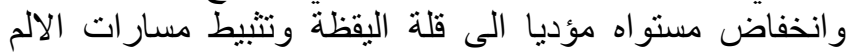

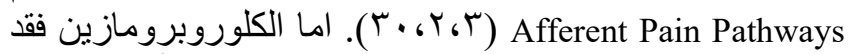

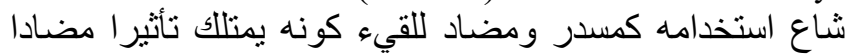

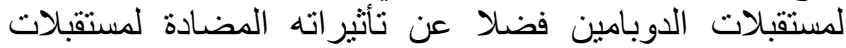

الهيتامين و الكولين (9 (19).

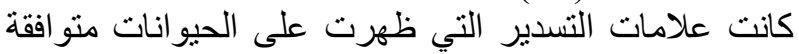

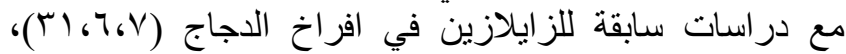

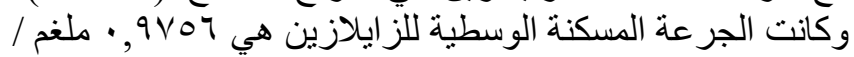

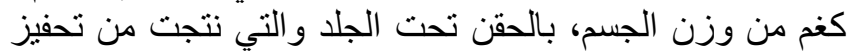

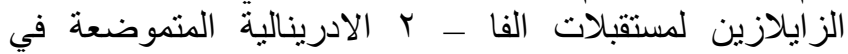

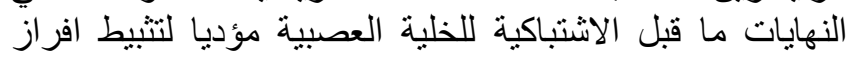

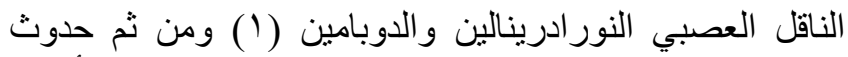

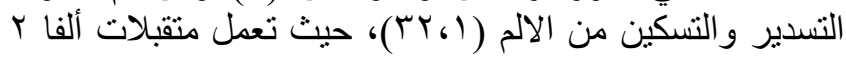

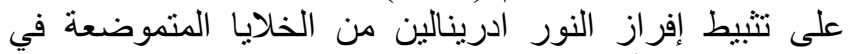
منطقة الموقع الأزرق في الدماغ، و هذا يؤدي إلى تسدير وتسكين النين 
18. Almeida RM, Valadao CAA, Reis RG. Premedication with amitraz or xylazine for anesthetic induction of cattle with ketamine. Ciencia Anim Brasileira. 2008;9(2):402-409.

19. Jaime Diaz. How drug influence behavior : a neurobehavioral approach. Englewood difts. N.T. Prentic hall. 1997; pp:285.

20. Lee I, Yamagishi N, Oboshi K, Yamada H. Antagonistic effects of intravenous or epidural atipamezole on xylazine-induced dorsolumbar epidural analgesia in cattle. Vet J. 2003;166:194-197.

ا Y. المشهداني، محمود بشير محمود. التأثير ات العصبية الدوائية للمديتومدين

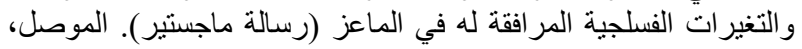

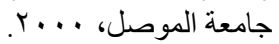

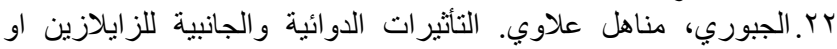

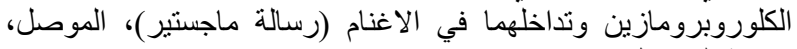

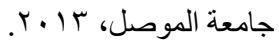

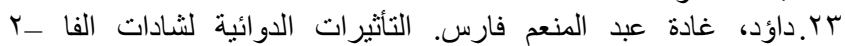

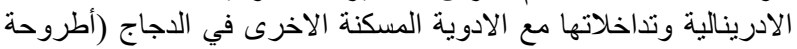

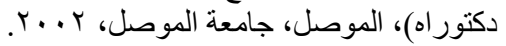

24. Dixon WJ. Efficient analysis of experimental observations. Annual Rev Pharmacol Toxicol. 1980;20:441-462.

25. Petrie A, Watson P. Statistics for veterinary and animal sciences. Oxford: Blackwell Science.1999;pp.90-140.

26. Katz MH. Bivariate statistics. In: Katz MH. editor. Study design and statistical analgesia. New York: Cambridge University press. 2006; pp.66-119.

27. Bluman AG. Elementary statistics, A step by step approach. $6^{\text {th }}$ ed. New York: McGraw-Hill companies. 2007.

28. Cleophas TJ, Zwinderman AH, Cleophas TF, Cleophas EP. Statistics applied to clinical Trials. $4^{\text {th }}$ ed. 2009. p.375-477.

29. Runyon RP. Non-parametric statistics: A contemporary approach. Massachusetts: Addison-Wesley publ Co. Reading. 1977;pp.2-217.

30. Cormack JR, Orme RM, Costello TG. The Role of Alpha-2 Agonists in Neurosurgery. J Clin Neuroscience. 2005;12:375-378.

31. Ruskoaho H, Karppanen H. Xylazine-induced sedation in chicks is inhibited by opiate receptor antagonists. Europ J Pharmacol. 1984;100(1):91-96.

32. Aantaa R. Alpha2-adrenoceptor agonists in human anesthesiology and intensive care. Proceedings of the $7^{\text {th }}$ World Congress of Veterinary Anesthesia. 20-23 Sept. 2000. Bern, Switzerland.

33. Short CE. Pain in animals. Las Vegas Nevada. Western Veterinary Conference. 1997;pp.5-100.
4. Gross ME. Tranquilizers, $\alpha 2$-Adrenergic agonists and therapeutics $8^{\text {th }}$ ed. Iowa State: University Press Ames. 2001;pp:299-324.

5. Varner J, Clifton KR, Poulos S, Borderson JR, Wyatt RD. Lack of efficacy of injectable ketamine with xylazine or diazepam for anesthesia in chicken. Lab. Anim.(NY). 2004;33(5):36-39.

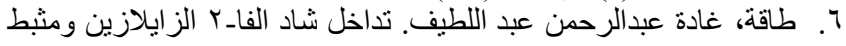

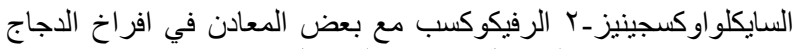

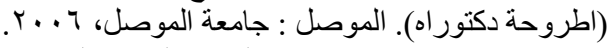

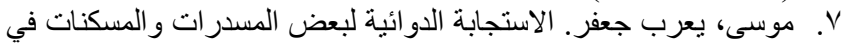

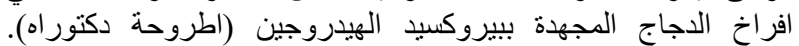

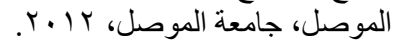

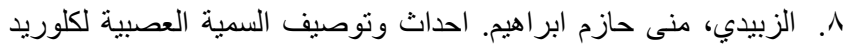

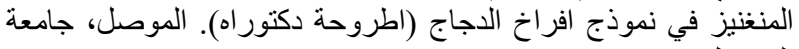

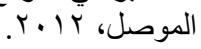

9. Hughan SC, Loose JM, Caddy DJ, Canny BJ, Tibrook AJ, Young IR. Combined xylazine and ketamine as an analgesic regimen in sheep. Aus Vet J. 2001;79(3):207-211.

10. Al-Qarawi AA. Immobilization (restraint) stress in desert sheep and goats, and the influence of pretreatment with xylazine or sodium betaine thereon. Pol J Vet Sci. 2005;8 (1):73-78.

11. Mama KR, Wagner AE, Steffey EP, Kollias-Baker C, Hellyer PW, Golden AE, Brevard LF. Evaluation of Xylazine and Ketamine for total intravenous anesthesia in horse. Amer $\mathrm{J}$ Vet Res. 2005;66(6):11002-7.

12. Lee I, Seo J, Son W, Gang S. Sedative and analgesic effects of intravenous xylazine and tramadol on horses. J Vet Sci. 2011;12(3):281-286.

13. Saifzadeh S, Pourjafar M, Dalir NB. Caudal extradural analgesia with lidocain, xylazine and a combination of lidocain and xylazine in the Iranian river buffalo. Bull Vet Inst Pulawy. 2007;51:285-288.

14. Cistola AM, Golder FJ, Centonze LA, Mckay LW, Levy JK. Anesthetic and physiologic effect of Tiletamine, Zolazepam, Ketamine and Xylazine combination (TKX) in cats undergoing surgical sterilization. J Feline Surg. 2004;6(5):297-303.

15. Thurmon JC, Tranquuilli WJ, Benson GJ. Lumb and Jones veterinary anesthesia. $3^{\text {rd }}$ ed. Baltimore: Williams and Wilkings. 1996; pp:185186,194

16. Gross ME. Tranquilizers, $\alpha 2$-adrenergic agonists, and related agents. In: Adams HR. editor. Vet Pharmacol Therap. $8^{\text {th }}$ ed. Iowa State: University Press. Ames; 2001;pp:299-324.

17. Papich MG. Saunders Handbook of Veterinary Drugs. $2^{\text {nd }}$ ed. North Carolina: State University. 2007. 